10. Pos W, Sorvillo N, Fijnheer R, et al. Residues Arg568 and Phe592 contribute to an antigenic surface for anti-ADAMTS13 antibodies in the spacer domain. Haematologica. 2011;96(11):1670-1677.

11. Jian C, Xiao J, Gong L, et al. Gain-of-function ADAMTS13 variants that are resistant to autoantibodies against ADAMTS13 in patients with acquired thrombotic thrombocytopenic purpura. Blood. 2012;119(16):3836-3843

12. Scully M, McDonald V, Cavenagh J, et al. A phase 2 study of the safety and efficacy of rituximab with plasma exchange in acute acquired thrombotic thrombocytopenic purpura. Blood. 2011;118 (7):1746-1753.

13. Hie M, Gay J, Galicier L, et al. Preemptive rituximab infusions after remission efficiently prevent relapses in acquired thrombotic thrombocytopenic purpura. Blood. 2014;124(2):204-210.

14. Westwood JP, Thomas M, Alwan F, et al. Rituximab prophylaxis to prevent thrombotic thrombocytopenic purpura relapse: outcome and evaluation of dosing regimens. Blood Adv. 2017;1(15):1159 1166

\title{
A new drug for an old concept: aptamer to von Willebrand factor for prevention of arterial and microvascular thrombosis
}

\author{
Agnès Veyradier ${ }^{1,2}$ \\ ${ }^{1}$ Hematology department, French National Reference Centre for Thrombotic Microangiopathies and von Willebrand disease, Hospital \\ Lariboisière, AP-HP.Nord and 'EA3518 Saint-Louis Research Institute, Paris University, Paris, France. \\ E-mail: AGNÈS VEYRADIER - agnes.veyradier@aphp.fr
}

doi:10.3324/haematol.2020.261081

$\mathrm{V}$ on Willebrand factor (VWF) is a large and complex multimeric glycoprotein essential for initiation of hemostasis after vascular injury. VWF is the mediator of platelet adhesion to the subendothelial collagen matrix and of platelet aggregation, especially at high shear rates of blood flow present in the microcirculation and stenotic arteries. ${ }^{1}$ Platelet adhesion involves specific sequences of the A1 domain of VWF (VWF-A1) and the platelet receptor glycoprotein Ib (GPIb). ${ }^{1}$ The adhesive properties of VWF are proportional to both the size of its multimers and their shear-induced unfolding, which respectively determine the number of available VWF-A1 and their swift from a cryptic to an exposed status able to bind platelet GPIb. ${ }^{2}$ Physiologically, in order to prevent the spontaneous binding of VWF to platelets, VWF multimeric distribution is regulated by a specific-cleaving protease, ADAMTS13 (a disintegrin and metalloproteinase with thrombospondin type 1 repeats, member 13). ${ }^{3}$ A defect in VWF (related to genetic mutations of VWF) causes a bleeding disorder named von Willebrand disease (VWD) while an excess of ultralarge multimers of VWF (UL VWF) (due to a severe deficiency in ADAMTS13 mostly mediated by specific auto-antibodies) causes a thrombotic microangiopathy called thrombotic thrombocytopenic purpura (TTP) ${ }^{4}$ In addition, the interaction of VWF-A1 with platelet GPIb also contributes to arterial thrombosis present in atherosclerotic cardiovascular disease (ACD). ${ }^{5}$ Consequently, inhibiting the binding of VWF to GPIb by specifically targeting VWF-A1, is a rational approach to decrease both arterial and microvascular thrombosis by preventing the formation of further VWF- and platelet-rich thrombi ${ }^{2}$ in both acute $\mathrm{ACD}^{5}$ and acute $T T \mathrm{P}^{3}$, respectively.

In the 1990-2000s, two classes of anti-VWF-A1 therapeutic agents were developed for this purpose. On one hand, a humanized single-variable domain immunoglobulin (Nanobody $\left.{ }^{\oplus}\right)^{6,7}$ has recently been approved and commercialized as caplacizumab (Cablivi ${ }^{\text {TM}}$ ) by Ablynx, a Sanofi company (Sanofi-Aventis, Paris, France) for the treatment of acute acquired TTP in adults, on the basis of positive results in phase II and III trials. ${ }^{8,9}$ On the other hand, several aptamers, consisting of single-stranded DNA or RNA oligonucleotides with a specific and stable three-dimensional shape able to recognize their target with high affinity and specificity, were developed and tested in animal models. ${ }^{10-12}$ However, only the historical anti-VWF-A1 aptamer, ARC1779, developed by Archemix (Cambridge, MA, USA), was investigated in $\mathrm{ACD}^{13-16}$ and $\mathrm{TTP}^{17-21}$ including limited phase II studies. ${ }^{16,19-21}$

In this issue of Haematologica, Sakai $\mathrm{K}$ and colleagues ${ }^{22}$ present the in vitro characterization of a recently developed novel aptamer to VWF-A1, TAGX-0004, ${ }^{23}$ using an elegant and extensive structural and functional investigation in both static and dynamic conditions (platelet aggregation, shear stress-induced platelet thrombus formation, study of binding to both wild-type VWF-A1 and 16 alanine-scanning VWF-A1 mutants using an electrophoresis mobility shift assay and surface plasmon resonance, and graphic analysis of three-dimensional (3D) structure models of VWF-A1). The authors show that, in vitro, TAGX-0004 is able to inhibit the binding of VWF-A1 to platelet GPIb better than the historical aptamer ARC1779 and as well as the Nanobody ${ }^{\oplus}$ caplacizumab. TAGX-0004 is thus presented as a new potential therapeutic option not only in acute TTP but also in various VWF-mediated thrombotic disorders such as acute coronary syndrome (ACS) and cerebral infarction.

In terms of the biochemical properties, the comparison of TAGX-0004 with ARC1779 is solid because those aptamers were used as both monovalent entities with no polyethylene glycol (PEG). The significantly higher affinity of TAGX0004 for VWF-A1 compared to ARC1779 is likely related to the presence of Ds, an artificial hydrophobic base, able to directly interact with a specific residue (F1366) within VWF-A1. Also, interestingly, the amino acid residues of VWF-A1 identified as binding sites for TAGX-0004 and ARC1779 by the current study did not totally overlap and some slight differences with the originally mapping of VWF-A1 binding sites for ARC1172/ARC1179 performed by Huang and colleagues ${ }^{24}$ were also observed. Regarding the similar in vitro affinity for VWF-A1 measured for both TAGX-0004 and caplacizumab, the authors mention that direct comparison of an affinity of monovalent entity with 


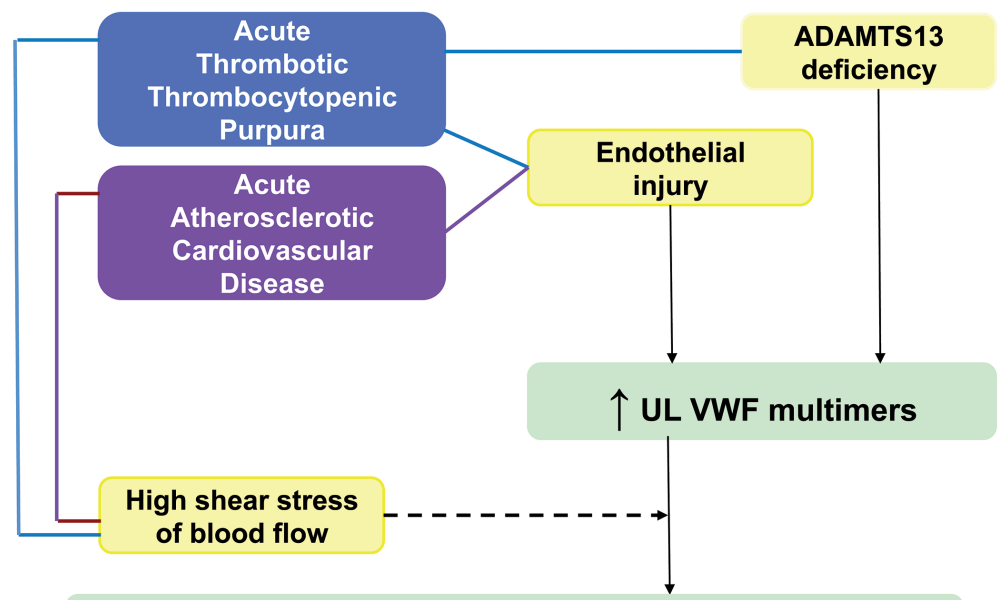

Unfolded UL VWF multimers exposing A1 domains

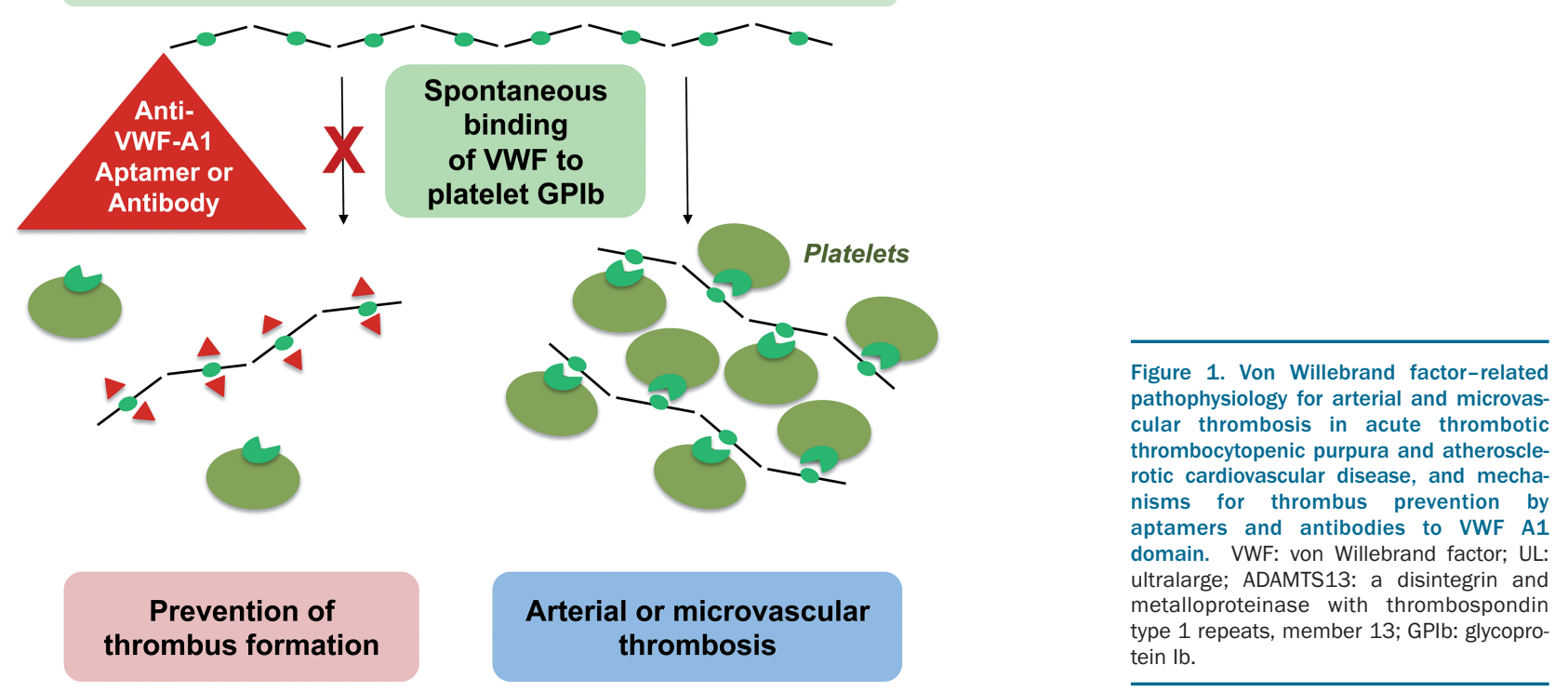

an avidity of bivalent entity is not straightforward, implying that this result should be considered with caution. Also, this study identified only a partial overlap of VWF-A1 binding sites for TAGX-0004 and caplacizumab, including however crucial amino acid residues (i.e., R1395 and R1399) found to be in common to all three: TAGX-0004, ARC1779 and caplacizumab.

To better consider preclinical and clinical trials' perspectives of this new anti-VWF-A1 aptamer, the historical background of both ARC1779 and caplacizumab is certainly important to recall. Since 2007, ARC1779 has been demonstrated to be efficient in vivo in a primate mode ${ }^{14}$ and to produce dose- and concentration-dependent inhibition of VWF activity and platelet function in a first-in-human evaluation led in healthy volunteers..$^{10}$ In addition, proof-of-concept of ARC1779 was provided in patients with VWD type $2 \mathrm{~B}^{25} \mathrm{a}$ genetic model of hyperadhesive VWF in which the mutated VWF-A1 exhibits a hyper-affinity for platelet GPIb leading to spontaneous binding to platelets and sometimes to thrombocytopenia. In ex vivo studies, ARC1779 effectively inhibited VWF activity in plasma samples of both TTP patients $^{18}$ and ACS patients. ${ }^{13,15}$ Some clinical experiences were first reported in three studies involving a total of 11 TTP patients. ${ }^{17,19,20}$ Then, two randomized, double-blind, placebo-controlled phase II studies were initiated in carotid endarterectomy ${ }^{16}$ and in acute TTP ${ }^{21}$ but both of them underwent premature closure due to cessation of funding. The first study ${ }^{16}$ was led in 36 patients and reported that intravenous ARC1779 reduced cerebral embolization postcarotid endarterectomy with however, a higher rate of peroperative bleeding complications in patients requiring surgery. The second study ${ }^{21}$ led in only seven TTP patients, showed that intravenous ARC1779 (loading dose followed by daily infusion performed after each therapeutic plasma exchange [TPE]) induced a suppression of VWF activity correlated to the plasma concentration of ARC1779, apparently decreased the number of TPE to achieve a normal platelet count and was well tolerated with no bleeding complications. Despite of the latter promising results in TTP, no ARC1779 trial has been published since 2012. The caplacizumab story is significantly different. However, originally, caplacizumab was under development for the prevention of thrombosis in ACS patients undergoing percutaneous coronary intervention ${ }^{6}$ but the development for this indication 
was discontinued in favor of TTP. After proof-of-concept in a pre-clinical baboon model of acquired TTP $^{7}$ in 2012, caplacizumab rapidly emerged as a new strategy of first-line treatment of acute TTP in association with TPE and immunomodulating agents. ${ }^{26,27}$ Caplacizumab was evaluated in two major multicenter, randomized, double-blind, placebo-controlled phase II $^{8}$ and phase III ${ }^{9}$ trials involving 36 and 72 TTP patients and published in 2016 and 2019, respectively: intravenous and subcutaneous caplacizumab showed superiority to placebo in terms of reduction of mortality, reduction of time to platelet recovery allowing an earlier stop of TPE, prevention of formation of further microthrombi and protection of organs from ischemia. Bleeding adverse event were common, affecting roughly half of the patients, but mostly mild or moderate and thus self-limited or resolved. ${ }^{8,9}$ Caplacizumab approval for acute TTP was obtained in 2018 in the EU and in 2019 in the US.

Here, the authors open the door to the new anti-VWFA1 aptamer as a promising molecule for future preclinical and clinical trials devoted to VWF-mediated thrombotic disorders, either the frequent ACD or the rare TTP.22 They emphasize that TAGX-0004 may get an antidote as already developed for other aptamers ${ }^{1,28}$ which may be a great advantage to control adverse bleeding events and thus to improve its main safety concern. In ischemic stroke and myocardial infarction, TAGX-0004 may be a rival of current antithrombotic and thrombolytic agents which are irreversible and associated with a significant risk of haemorrhage. ${ }^{5}$ In addition, in contrast to antiplatelet drugs which bypass platelet adhesion and inhibit only platelet aggregation, TAGX-0004 is able to prevent the initial step of thrombus formation by inhibiting platelet adhesion and, as a consequence, also platelet aggregation. In acute TTP, TAGX-0004 may appear as a rival of caplacizumab. However, considering the complexity of TTP as a rare and life-threatening disease, several questions require specific attention during preclinical and clinical studies. First, acute TTP management relies on a mandatory concomitant use of first-line multiple targeted therapies ${ }^{26,27}$ i.e., TPE and future recombinant ADAMTS13 as the replacement therapy for severely deficient ADAMTS13, steroids and rituximab as immunomodulators against anti-ADAMTS13 autoantibodies and now caplacizumab as an inhibitor of VWF-A1 preventing the adhesion of UL VWF to platelets. Thus, potential interactions of aptamers with ADAMTS13-replacing products and immunomodulators are unknown so far. Second, whether TAGX-0004 is able, like caplacizumab, to form a complex with VWF which clearance leads to a partial decrease of VWF antigen and coagulation factor VIII beneficial for the thrombotic atmosphere of TTP, is also unknown. Third, the superiority of TAGX-0004 to caplacizumab in terms of the bleeding risk remains to be further investigated as i) caplacizumab-associated bleeding events are mostly moderate and easily resolved, ${ }^{8,9}$ and ii) the potential TAGX-0004 antidote constitutes an extra drug that may exhibit specific adverse events. Fourth, even if the in vivo half-life of TAGX-0004 is upgraded by a mini-hairpin DNA structure conferring resistance to degradation by nucleases, ${ }^{22}$ it has to be compatible with a daily regimen adjusted to TPE. Fifth, as aptamers also have the capacity to be used as diagnostic reagents (their com- bined therapeutics and diagnostics potential being summarized as "theranostic"), ${ }^{28}$ the potential interference of TAGX-0004 with ADAMTS13 biologic assays crucial for therapy-driven monitoring in acute TTP ${ }^{28}$, should also be a point of attention.

Today, aptamer technology remains one step behind the humanized monoclonal antibody research and development, mostly because antibodies biotech industry's financial investment has been highly prioritized for the last three decades. In 2020, only one aptamer targeted to vascular endothelial growth factor (Macugen/Pegaptanib sodium) has gained approval by the US Food and Drug Aminsitration for patients with age-related macular degeneration and few aptamers have successfully entered clinical trials for different therapeutic indications. ${ }^{28}$ However, considering their advantages over antibodies (low price, small size, easy production) together with the efforts made to overcome their limitations (improvement of stability, target affinity, in vivo retention and corrective approaches to potential unmethylated 2'-deoxycytidinephosphate-2'-guanine toxicity), ${ }^{28}$ aptamers begin to slowly penetrate niche markets and bring promising therapeutic perspectives.

\section{References}

1. Matsushita T, Meyer D, Sadler JE. Localization of von Willebrand factor-binding sites for platelet glycoprotein Ib and botrocetin by chargedto-alanine scanning mutagenesis. J Biol Chem. 2000;275(15):1104411049

2. De Meyer SF, Vanhoorelbeke K, Ulrichts H, et al. Development of monoclonal antibodies that inhibit platelet adhesion or aggregation as potential anti-thrombotic drugs. Cardiovasc Hematol Disord Drug Targets. 2006; 6(3):191-207.

3. South K and Lane DA. ADAMTS13 and von Willebrand factor: a dynamic duo. J Thromb Haemost. 2018;16(1):6-18.

4. Sadler JE. Pathophysiology of thrombotic thrombocytopenic purpura. Blood. 2017;130(10):1181-1188.

5. Montalescot G, Philippe F, Ankri A, et al. Early increase of von Willebrand factor predicts adverse outcome in unstable coronary artery disease: beneficial effects of enoxaparin. French Investigators of the ESSENCE Trial. Circulation. 1998;98(4):294-299.

6. Ulrichts H, Silence K, Schoolmeester A, et al. Antithrombotic drug candidate ALX-0081 shows superior preclinical efficacy and safety compared with currently marketed antiplatelet drugs. Blood. 2011;118(3):757-765.

7. Callewaert F, Roodt J, Ulrichts H, et al. Evaluation of efficacy and safety of the anti-VWF nanobody ALX-0681 in a preclinical baboon model of acquired thrombotic thrombocytopenic purpura. Blood. 2012;120(17): 3603-3610.

8. Peyvandi F, Scully M, Kremer Hovinga JA, et al. Caplacizumab for acquired thrombotic thrombocytopenic purpura. $N$ Engl J Med. 2016;374(6):511-522.

9. Scully M, Cataland SR, Peyvandi F, et al. Caplacizumab treatment for acquired thrombotic thrombocytopenic purpura. N Engl J Med. 2019;380(4):335-346

10. Gilbert JC, DeFeo-Fraulini T, Hutabarat RM, et al. First-in-human evaluation of anti von Willebrand factor therapeutic aptamer ARC1779 in healthy volunteers. Circulation. 2007;116(23):2678-2686.

11. Nimjee SM, Lohrmann JD, Wang $\mathrm{H}$, et al. Rapidly regulating platelet activity in vivo with an antidote controlled platelet inhibitor. Mol Ther. 2012;20(2):391-397.

12. Nimjee SM, Dornbos III D, Pitoc GA, et al. Preclinical development of a vWF aptamer to limit thrombosis and engender arterial recanalization of occluded vessels. Mol Ther. 2019;27(7):1228-1241.

13. Spiel AO, Mayr FB, Ladani N, et al. The aptamer ARC1779 is a potent and specific inhibitor of von Willebrand Factor mediated ex vivo platelet function in acute myocardial infarction. Platelets. 2009;20(5):334-340

14. Diener JL, Daniel Lagassé HA, Duerschmied D, et al. Inhibition of von Willebrand factor-mediated platelet activation and thrombosis by the anti-von Willebrand factor A1-domain aptamer ARC1779. J Thromb 
Haemost. 2009;7(7):1155-1162.

15. Arzamendi D, Dandachli F, Théorêt JF, et al. An anti-von Willebrand factor aptamer reduces platelet adhesion among patients receiving aspirin and clopidogrel in an ex vivo shear-induced arterial thrombosis. Clin Appl Thromb Hemost. 2011;17(6):E70-78.

16. Markus HS, McCollum C, Imray C, et al. The von Willebrand inhibitor ARC1779 reduces cerebral embolization after carotid endarterectomy: a randomized trial. Stroke. 2011;42(8):2149-2153.

17. Knöbl P, Jilma B, Gilbert JC, et al. Anti-von Willebrand factor aptamer ARC1779 for refractory thrombotic thrombocytopenic purpura. Transfusion. 2009;49(10):2181-2185.

18. Mayr FB, Knöbl P, Jilma B, et al. The aptamer ARC1779 blocks von Willebrand factor-dependent platelet function in patients with thrombotic thrombocytopenic purpura ex vivo. Transfusion. 2010;50(5): 1079-1087.

19. Jilma-Stohlawetz P, Gorczyca ME, Jilma B, et al. Inhibition of von Willebrand factor by ARC1779 in patients with acute thrombotic thrombocytopenic purpura. Thromb Haemost. 2011;105(3):545-552.

20. Jilma-Stohlawetz P, Gilbert JC, Gorczyca ME, et al. A dose ranging phase I/II trial of the von Willebrand factor inhibiting aptamer ARC1779 in patients with congenital thrombotic thrombocytopenic purpura. Thromb Haemost. 2011;106(3):539-547.

21. Cataland SR, Peyvandi F, Mannucci PM, et al. Initial experience from a double-blind, placebo-controlled, clinical outcome study of ARC1779 in patients with thrombotic thrombocytopenic purpura. Am J Hematol. 2012;87(4):430-432.
22. Sakai K, Someya T, Harada K, Yagi H, Matsui T, Matsumoto M. Novel aptamer to von Willebrand factor A1 domain (TAGX-0004) shows total inhibition of thrombus formation superior to ARC1779 and comparable to caplacizumab. Haematologica. Haematologica. 2020;105 (11):2631-2638

23. Matsunaga KI, Kimoto M, Hirao I, et al. High-affinity aptamer generation targeting von Willebrand factor A1-domain by genetic alphabet expansion for systematic evolution of ligands by exponential enrichment using two types of libraries composed of five different bases. J Am Chem Soc. 2017;139(1):324-334.

24. Huang RH, Fremont DH, Diener JL, Schaub RG, Sadler JE. A structural explanation for the antithrombotic activity of ARC1172, a DNA aptamer that binds von Willebrand factor domain A1. Structure. 2009;17(11):1476-1484.

25. Jilma-Stohlawetz P, Knöbl P, Gilbert IC, et al. The anti-von Willebrand factor aptamer ARC1779 increases von Willebrand factor levels and platelet counts in patients with type $2 \mathrm{~B}$ von Willebrand disease. Thromb Haemost. 2012;108(2):284-290

26. Joly BS, Vanhoorelbeke K, Veyradier A. Understanding therapeutic tar gets in thrombotic thrombocytopenic purpura. Intensive Care Med. 2017:43(9):1398-1400.

27. Coppo P, Cuker A, George JN. Thrombotic thrombocytopenic purpura: toward targeted therapy and precision medicine. Res Pract Thromb Haemost. 2018;3(1):26-37.

28. Kaur H, Bruno JG, Kumar A, Sharma TK. Aptamers in the therapeutics and diagnostics pipelines. Theranostics. 2018;8(15):4016-4032. 